\title{
Computation of internal stress resultants in beam elements with constrained torsional warping
}

\author{
Koen Dwarshuis, Marijn Nijenhuis, Ronald Aarts, Marcel Ellenbroek, Dannis Brouwer \\ Faculty of Engineering Technology \\ University of Twente \\ P.O. Box 217, 7500 AE Enschede, The Netherlands \\ [k.s.dwarshuis, m.nijenhuis, r.g.k.m.aarts, m.h.m.ellenbroek, d.m.brouwer]@utwente.nl
}

\begin{abstract}
The computation of stress in beam elements requires the internal stress resultants like axial force and bending moments. For displacement-based beam elements, these resultants can be obtained 1) based on equilibrium, 2) consistent to the constitutive law or 3) based on load interpolation functions. Although the methods give similar results in case of small deformation, the discrepancies in case of large deformation are significant. This paper shows that the method based on equilibrium gives the most accurate results.

Torsion of beam elements causes warping of the cross section. This warping is constrained at clamped ends of beam elements, causing extra stress. This paper shows a method to accurately obtain the corresponding internal stress resultants, i.e. the Saint-Venant torsion moment and bimoment.
\end{abstract}

Keywords: Beam, stress, internal stress resultants, Vlasov beam theory, flexures.

\section{INTRODUCTION}

Structures are often analyzed using beam-elements. This paper is motivated by flexure mechanisms in particular, where each leaf spring can be modelled by multiple serial connected beam elements [1-5]. Fig. 1 shows an example. Stress in mechanisms that are modelled by beamelements can be computed in three steps, see Fig. 1:

1. The displacements, deformations and reaction forces on each beam element are computed based on the stiffness relations;

2. For each beam-element the internal stress resultants are computed at a finite number of points along the beam axis. These stress resultants are quantities like the axial force and bending moments.

3. The stress-distribution on the cross section is computed based on the internal stress resultants. Formulas for this can be found in standard text books [6].

This paper focusses on step 2, the computation of internal stress resultants for three-dimensional beams undergoing large deformation. Firstly by comparing 3 different methods to compute five of the internal stress resultants and secondly by proposing an accurate method to interpolate the internal stress resultants related to torsion.

The internal stress resultants can be determined from equilibrium equations or be computed consistent with the constitutive law. These two methods give a different result. For small planar elastic deformations though, it was concluded that the discrepancies between these methods are small [7-9].

However, the deformation in a beam element can be large. Moreover, by using beam elements that are accurate for larger deformation, less serial connected beam elements are required to model a single leafspring accurately, and this increases the computational efficiency. A significant 
amount of literature is published on the modelling of stiffness of beam elements for large deformation. An overview can be found in [10]. The use of these beam models urges for an accurate method to obtain internal stress resultants in case of large deformation.

Two specific beam formulations are used in this paper, a $2^{\text {nd }}$ order formulation [11] and a $3^{\text {rd }}$ order formulation [12]. The $3^{\text {rd }}$ order formulation is based on the Hellinger-Reissner principle, which implies that interpolation functions for both load and displacement fields are used. The load interpolation functions can be used to obtain the internal stress resultants. This is the third method that is used in this paper for comparison.

Torsion of a beam generally causes warping of the cross section. This warping however is constrained at the clamped ends of the beam element and this can cause significantly extra stiffness and stress $[13,14]$. This effect can be included in a beam element by two extra deformation modes for the torsion, which is also applied in the used formulations [11, 12]. The correct computation of the corresponding stress requires two internal stress resultants, namely the bimoment and the Saint-Venant torsion. These stress resultants however cannot be derived very accurately by the three previously mentioned methods. This paper presents a more suitable method to obtain the bimoment and Saint-Venant torsion moment.

The results in this paper are derived for the case of flexure mechanisms, using beam elements with thin rectangular cross section, in which the torsional warping is explicitly modelled. However, the results also apply to most other applications, beam formulations and different cross sectional shapes.

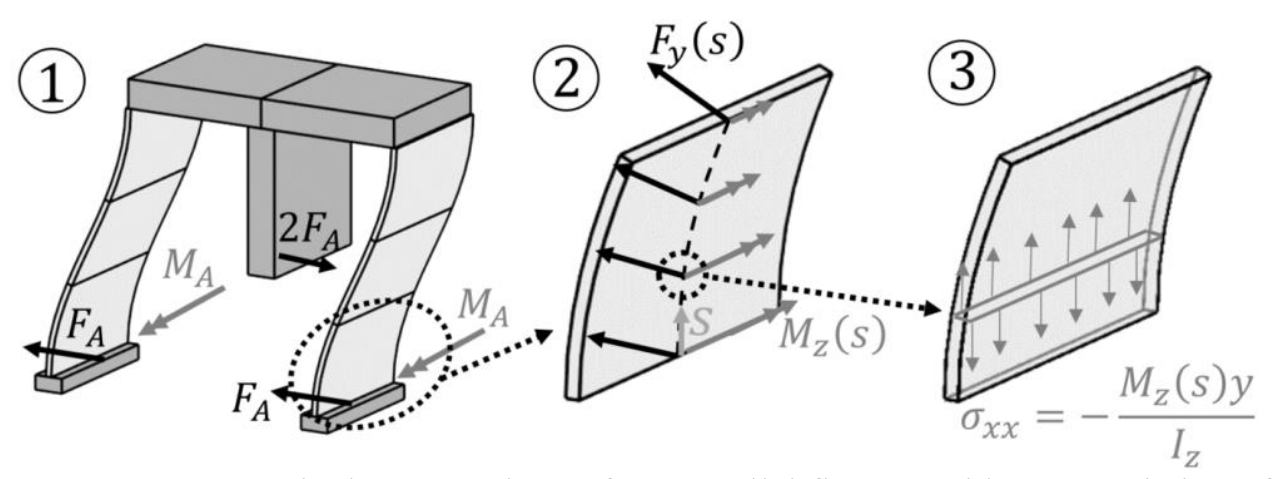

Figure 1. Steps to obtain stress, shown for a parallel flexure guidance consisting of two flexures (each modelled by three beam elements) and a connecting rigid part, 1) compute forces and displacements of a mechanism, 2) compute for each beam element the internal stress resultants 3 ) compute the stress distribution on the cross section

\section{METHOD}

This section shows how the internal stress resultants can be obtained based on the results of step 1 of Fig. 1. These results are visualized in Fig. 2: the positions $\left(\boldsymbol{r}_{p} \boldsymbol{r}_{q}\right)$ and orientations $\left(\boldsymbol{R}_{p}, \boldsymbol{R}_{q}\right)$ of both nodes of the beam-element, and the forces $\left(\boldsymbol{F}_{p}, \boldsymbol{F}_{q}\right)$, moments, $\left(\boldsymbol{M}_{p}, \boldsymbol{M}_{q}\right)$ and bimoments $\left(B_{p}, B_{q}\right)$ at both nodes. Based on the nodal positions, orientations and the mode-shapes of the element, the local displacements, $\left(u_{x}(s), u_{y}(s), u_{z}(s)\right)$ and local rotations $\left(\phi_{x}(s), \phi_{y}(s), \phi_{z}(s)\right)$ can be obtained. Here $s$ is the axial coordinate from 0 to the undeformed length $L_{0}$. Using these local displacements, the global positions $(\boldsymbol{r}(s))$ and orientations $(\boldsymbol{R}(s))$ inside the element can also be found.

Seven internal stress resultants should be obtained: section 2.1 presents three methods to obtain the axial force, shear forces in the local $y$-direction and $z$-direction and the bending moment around the local $y$-axis and $z$-axis. Section 2.2 explains three methods to obtain the Saint-Venant torsion moment and the bimoment. 


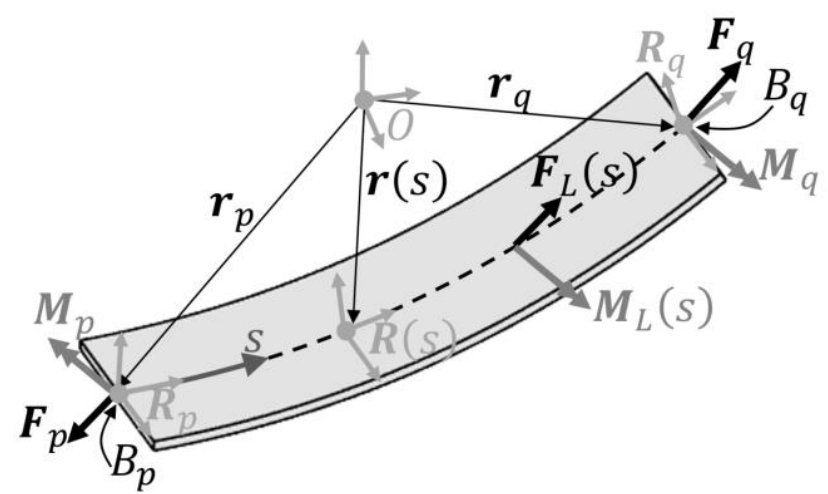

Figure 2. Beam-element, showing the forces, positions and orientations for both nodes and internally. Reference frame $O$ is the global reference frame.

\subsection{Internal stress resultants for extension, shear and bending}

Method A1 - Equilibrium. The internal stress resultants can be found based on equilibrium, using the values at both nodes:

$$
\boldsymbol{F}_{L}(s)=\boldsymbol{R}^{T}(s) \boldsymbol{F}_{q}, \boldsymbol{M}_{L}(s)=\boldsymbol{R}^{T}(s)\left(\boldsymbol{M}_{q}+\left(\boldsymbol{r}(s)-\boldsymbol{r}_{q}\right) \times \boldsymbol{F}_{q}\right)
$$

where the subscript $L$ emphasizes that it is expressed in the local reference frame $\boldsymbol{R}(s)$. The local force is composed of the axial force and the 2 shear forces and the moment is composed of the total torsion moment and the 2 bending moments:

$$
\boldsymbol{F}_{L}(s)=\left\{\begin{array}{l}
F_{x}(s) \\
F_{y}(s) \\
F_{z}(s)
\end{array}\right\}, \boldsymbol{M}_{L}(s)=\left\{\begin{array}{l}
M_{x}(s) \\
M_{y}(s) \\
M_{z}(s)
\end{array}\right\} .
$$

Method A2 - Constitutive law. The internal stress resultants are directly related to the derivatives of the local displacements:

$$
\begin{array}{ll}
F_{x}(s)=E A u_{x}^{\prime}(s) & \\
F_{y}(s)=G A \kappa_{y}\left(u_{y}^{\prime}(s)-\phi_{z}(s)\right), & M_{y}(s)=E I_{y} \phi_{y}^{\prime}(s), \\
F_{z}(s)=G A \kappa_{z}\left(u_{z}^{\prime}(s)+\phi_{y}(s)\right), & M_{z}(s)=E I_{z} \phi_{z}^{\prime}(s),
\end{array}
$$

where ( $)^{\prime}$ defines a derivative to coordinate $s . E$ is the elasticity modulus of the material and $G$ the shear modulus, $A$ is the cross sectional area, $\kappa$ the shear correction factor according to Cowper $[15]$ and $I_{y}$ and $I_{z}$ are the second moments of area.

Method A3 - Load interpolation functions. The third order beam element is derived based on the Hellinger-Reissner principle. This implies that it is derived based on a combination of load interpolation functions and displacement interpolation functions of which the corresponding coordinates are computed in step 1 of Fig 1. These load interpolation functions (see eq. 24 of ref. [12]) give a direct estimation for the required internal stress resultants.

\subsection{Saint-venant torsion moment and bimoment}

Torsion causes warping of the cross section. At the clamped ends of a beam this warping is constrained, resulting in additional strain energy storage, which causes extra stiffness and stress. The internal stress resultant related to warping is the bimoment, $B$. The shear stress is related to the Saint-Venant torsion moment, $T_{x}$. According to Vlasov torsion theory [14] the total torsion moment is composed of the Saint-Venant torsion moment and the derivative of the bimoment:

$$
T_{x}(s)+B^{\prime}(s)=M_{x}(s),
$$

Below, three methods are given to compute the Saint-Venant torsion moment and the bimoment. 
Method B1 - Interpolation. The bimoment is available at both nodes such that the bimoment can be obtained by a linear interpolation between these two values:

$$
B(s)=-B_{p} \frac{L-s}{L}+B_{q} \frac{s}{L}
$$

The Saint-Venant torsion moment is not available at the nodes so it cannot be obtained by interpolation. The Saint-Venant torsion moment can however be approximated by the total torsion moment $T_{x}(s) \approx M_{x}(s)$, which is a good approximation far from the clamped ends. This total torsion moment $M_{x}(s)$ can be computed based on equilibrium, see method A1, eq. (2).

Method B2 - Constitutive law. The Saint-Venant torsion moment and bimoment are directly related to derivatives of the torsion angle:

$$
T_{x}(s)=G I_{t} \phi_{x}^{\prime}(s), \quad B(s)=-E I_{\omega} \phi_{x}^{\prime \prime}(s)
$$

where $I_{t}$ is Saint-Venant's torsion constant and $I_{\omega}$ is Vlasov's warping constant [14].

Method B3 - ODE. The Saint-Venant torsion moment and the bimoment can be solved based on the differential equation in eq. (4). According to eq. (6) the Saint-Venant torsion moment and bimoment are related like:

$$
B(s)=-\frac{E I_{\omega}}{G I_{t}} T_{x}^{\prime}(s)
$$

Substituting this result into eq. (4) gives the ordinary differential equation (ODE):

$$
T_{x}-\frac{E I_{\omega}}{G I_{t}} T_{x}^{\prime \prime}=M_{x}(s)
$$

To solve for $T_{x}$ a homogeneous and a particular solution have to be obtained.

For the particular solution $M_{x}(s)$ is approximated by a $4^{\text {th }}$ order polynomial. This is done by first approximating the internal forces on the undeformed element (the orange, dotted line in Fig. 3a). The torsion $M_{x}^{*}(s)$ and the shear force $F_{z}^{*}(s)$ are linearly interpolated between their values on the nodes. The bending moment $M_{y}^{*}(s)$ is approximated by a second order polynomial that corresponds to the moments on the nodes and satisfies $M_{y}^{* \prime \prime}(s)=F_{z}^{* \prime}(s)$. Then, using equilibrium considerations, the total torsion moment at the deformed line (black dotted line in Fig. 3a) is computed by:

$$
M_{x}^{(4 t h)}(s)=M_{x}^{*}(s)+M_{y}^{*}(s) \cdot \phi_{z}(s)-F_{z}^{*}(s) \cdot u_{y}(s)
$$

The resulting relation is fourth order, assuming the displacement $u_{y}(s)$ to be a third order polynomial which is common in beam elements. Note that this equation neglects displacements in the z-direction as the shown beam element is very stiff in this direction. However, displacements in the z-direction could be accounted for in similar way as for the $y$-direction by including the term $-M_{z}^{*}(s) \cdot \phi_{y}(s)+F_{y}^{*}(s) \cdot u_{z}(s)$. Having a polynomial expression for $M_{x}(s)$, the particular solution $T_{x}^{(P)}$ of the ODE is easily obtained. The corresponding bimoment can be obtained by using eq. (7). For a constant total torsion moment, the solution is $T_{x}^{(P)}(s)=M_{x}$, $B^{(P)}(s)=0$. This indicates that the particular solution describes the bimoment due to a variation of the total torsion moment over the axial coordinate.

The homogeneous solution describes the effects at the boundaries of a leafspring where the warping is usually fully constrained or completely released. The homogeneous solution of the ODE is:

$$
T_{\chi}^{(H G)}\left(s_{L F}\right)=C_{1} \cosh \left(\lambda s_{L F}\right)+C_{2} \sinh \left(\lambda s_{L F}\right), \lambda=\sqrt{G I_{t} / E I_{\omega}}
$$

where $s_{L F}$ is the axial coordinate for a whole leaf spring, from 0 to the undformed length $L_{L F}$. The corresponding bimoment is, according to eq. (7):

$$
B^{(H G)}\left(s_{L F}\right)=-\sqrt{\frac{E I_{\omega}}{G I_{t}}}\left(C_{1} \sinh \left(\lambda s_{L F}\right)+C_{2} \cosh \left(\lambda s_{L F}\right)\right)
$$

The constants $C_{i}$ are computed based on the end-conditions of a full leafspring. For a clamped 
end we have the boundary condition $T_{x}=0$ and for a free end we have the condition $B=0$ (see Fig. 3b). So the particular solution was computed for each beam element individually, where the homogeneous solution is computed for a whole leafspring at once.

Note that as this method does not use the bimoment at the nodes that was computed in step 1 of Fig 1, it can also be used with beam elements that do not have warping modes.
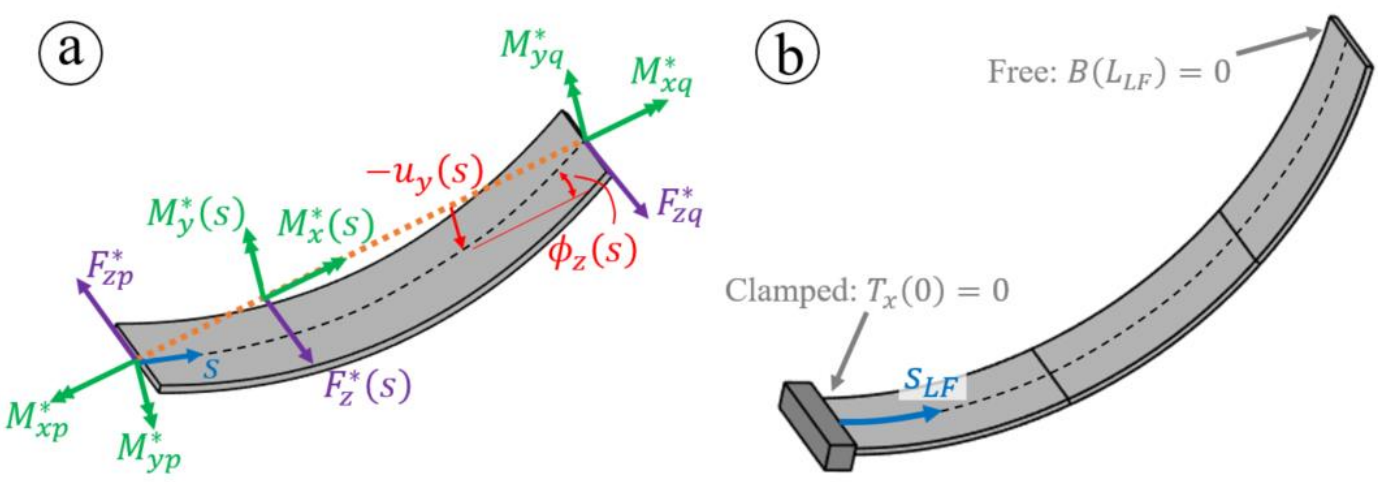

Figure 3. a) The particular solution is obtained using an estimation of the total torsion moment, which is derived based on an approximation of the forces on the undeformed line $M_{x}^{*}(s), M_{y}^{*}(s)$ and $F_{z}^{*}(s)$, and its deflection, $u_{y}(s), \phi_{z}(s)$. b) The homogeneous solution is based on the end-points of a whole leafspring

\section{RESULTS}

This section applies the methods to investigate which method is most accurate. A $2^{\text {nd }}$ order beam model [11] and a $3^{\text {rd }}$ order model [12] are used, with slightly different deformation modes as derived in appendix A of [16]. A rectangular beam with the following dimensions and material properties is used: length: $L=100 \mathrm{~mm}$, width: $w=10 \mathrm{~mm}$, thickness: $t=0.3 \mathrm{~mm}$, material elasticity: $E=200 \mathrm{GPa}$, Poisson ratio: $v=0.3$. Vlasov's warping constant is computed as $I_{\omega}=$ $w^{3} t^{3} / 144$, see $[17,18]$. The left side of the beam is fixed to the ground, the torsional warping at both sides is constrained and the right side is subjected to six different loading conditions, visualized in Fig. 4.
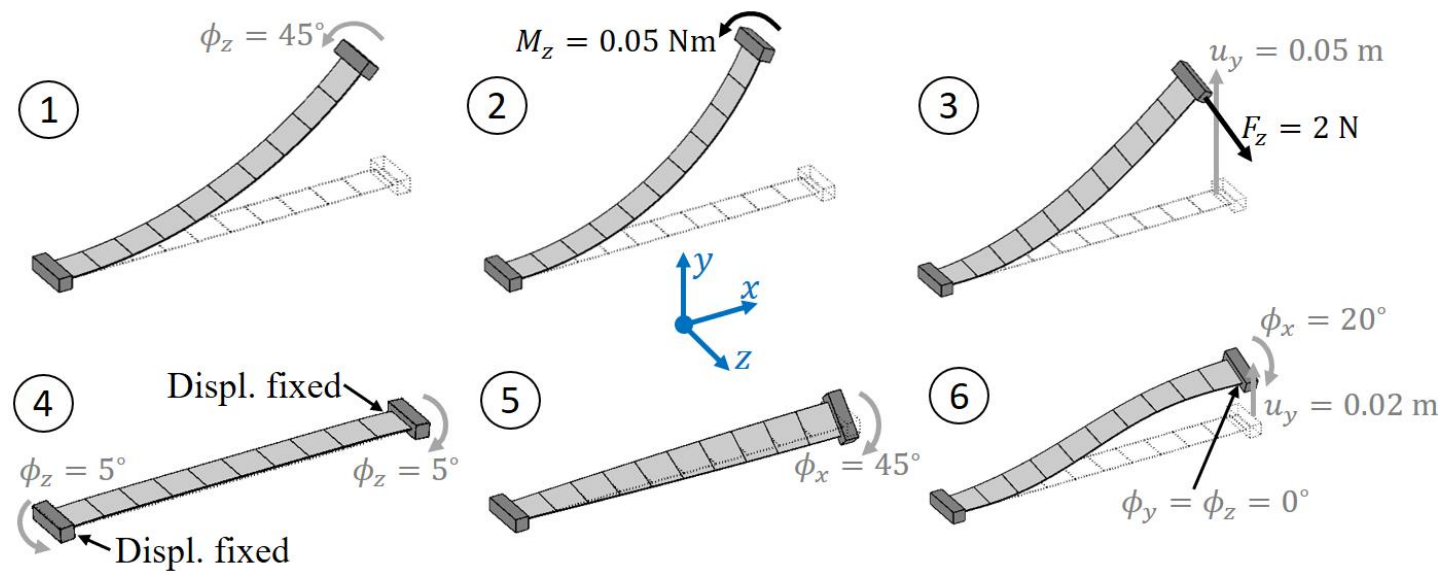

Figure 4. Load cases of the leaf spring, modelled by 10 beam elements. The left side is always completely fixed, except for case 4 . 1) bending rotation, 2) applied bending moment, 3) bending displacement in combination with a shear force, 4) the displacement of both ends is fixed and both ends are rotated to create a coupling between the axial and bending direction, 5) torsion, 6) torsion in combination with bending to create a significantly varying torsion over the axial coordinate. 

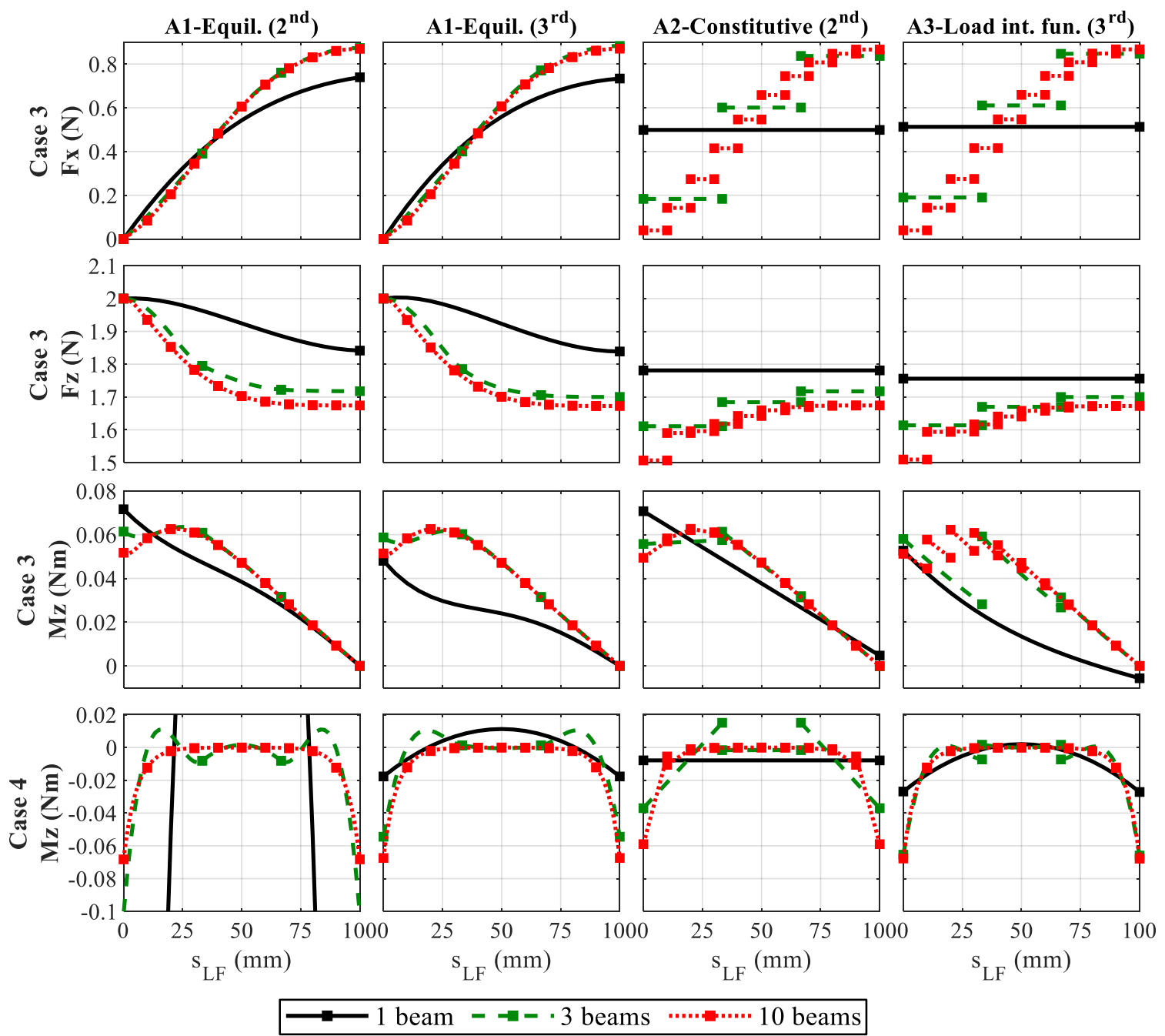

Figure 5. Resulting internal stress resultants as function of the axial coordinate $S_{L F}$ for two loading cases shown in Fig. 4. The leaf spring is modelled with 1, 3 and 10 beam elements. Method A1 is applied with the $2^{\text {nd }}$ order and with the $3^{\text {rd }}$ order beam element. Method A2 is only applied with the $2^{\text {nd }}$ order beam element (the results with the $3^{\text {rd }}$ order element were worse in general). Method A3 can only be applied with the $3^{\text {rd }}$ order element.

Case 1 and 2 are simple, the only nonzero internal stress resultant is the bending moment around the z-axis which is constant. For these two cases all methods give the exact result. Fig. 5 shows results for case 3 and 4 to compare methods A1, A2 and A3. Some observations are:

- The differences between the results of the three methods are significant if few beam elements are used.

- The results show that all methods converge to the same result if many beam elements are used in series, indicating that all methods converge to the exact solution. One exception on this observation is in the shear force $F_{z}$ where method A2 and A3 give fundamentally wrong results. Appendix A explains this and shows that the resulting relative error in the final stress is small.

- Method A1 converges the most rapidely to the exact solution when using more beam elements. The most imporantant reason for this is that this method accounts for local rotations of the cross section, i.e. the vector with internal foces $\boldsymbol{F}_{L}(s)$ and the vector with internal moments $\boldsymbol{M}_{L}(s)$ is rotated according to the orientation of the cross section $\boldsymbol{R}(s)$, see eq. (1).

- The internal stress resultants obtained by method A1 are continuous between the elements, where this is not the case for the methods A2 and A3. 
- The $3^{\text {rd }}$ order beam element gives generally better results than the $2^{\text {nd }}$ order beam element, especially in case 4.

Fig. 6 shows results of torsion (method B1-B3). The following observations are made:

- The bimoment on the beam nodes of case 5 is perfectly computed by the $3^{\text {rd }}$ order beam element, but method B1 still gives a quite bad estimation inside the elements because of the linear interpolation. Method B2 also gives significant errors, even with 10 beam elements the bimoment at both ends is $40 \%$ off. Method B3 gives a perfect result for case 5 , even with only one beam element.

- In case 6 the total torsion moment varies over the axial coordinate and therefore the bimoment at the nodes is not accurately approximated by the $2^{\text {nd }}$ order and $3^{\text {rd }}$ order beam element if only 1 or 3 beam elements are used. Therefore method B1 gives a bad estimation of the internal bimoment. Method B3 gives a relatively accurate result, even with only 1 beam element.

- The Saint-Venant torsion moment in case 6 is in method B1 approximated by the total torsion moment. Internally this approximation is quite good (for 3 or more beam elements) but not at both ends of the leaf spring. In method B2 clearly a lot of beam elements are required for an accurate estimation of the Saint-Venant torsion moment. Method B3 gives an accurate result with 3 or more beam elements.
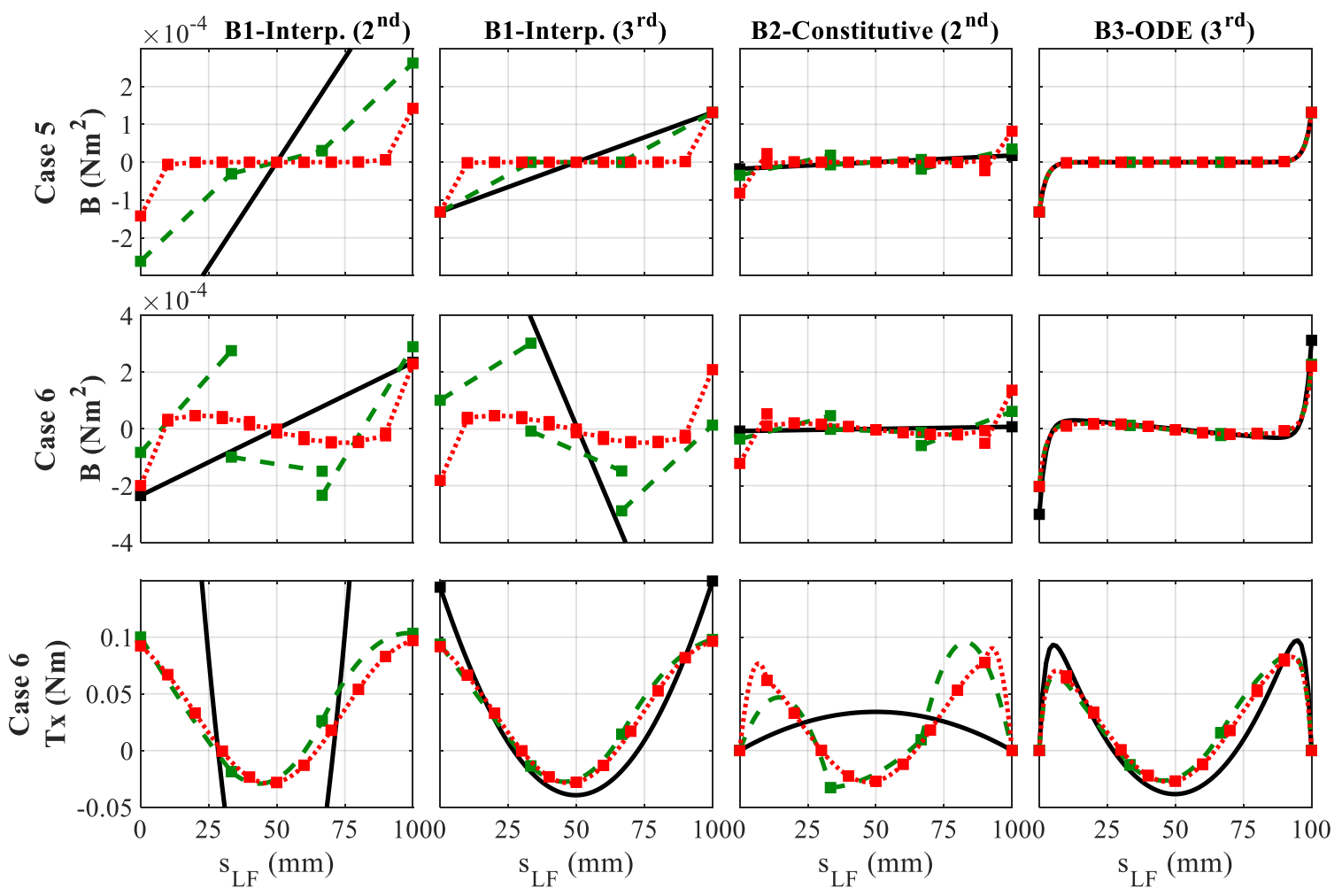

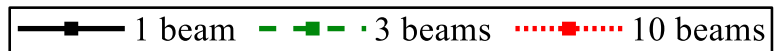

Figure 6. Resulting internal bimoment and Saint-Venant torsion moment as function of the axial coordinate $s_{L F}$. The leaf spring is modelled with 1,3 and 10 beam elements. Results are given for methods B1-B3 based on results of the $2^{\text {nd }}$ order or $3^{\text {rd }}$ order beam element. 
In summary, method $\mathrm{A} 1$ gives more accurate results than method $\mathrm{A} 2$ and $\mathrm{A} 3$. The most important reason is that it is easy to evaluate the nonlinear equilibrium-equation (see eq. (1)), therefore method A1 accounts for the effect of local displacements and rotations of the cross sections on the equilibrium. Method A2 and A3 use interpolation functions that are based on equilibrium in the undeformed state.

Method B3 gives the most accurate results for torsion. Similar to method A1, this method obtains an accurate estimation of the total torsion moment based on equilibrium and the local displacements of the beam. Based on this estimation the Saint-Venant torsion moment and the bimoment can be obtained accurately.

As this reasoning is not limited to the used beam dimensions and loading conditions, method A1 and $\mathrm{B} 3$ will be the most accurate for displacement based elements in general.

\section{CONCLUSIONS}

The computation of stress in beam elements requires the internal stress resultants to be obtained. These resultants can be obtained by different methods, which result in significant different results in case of large deformation. Three methods were compared to obtain the internal stress resultants for extension, shear and bending. The method based on equilibrium equations gives the most accurate results.

Three other methods are proposed to obtain the internal stress resultants related to torsion, i.e. the Saint-Venant torsion moment and the bimoment. It was found that these resultants can be obtained accurately based on the solution of the differential equation that relates the total torsion moment, the Saint-Venant torsion moment and the bimoment.

The results indicate that a right choice of the method to obtain the internal stress resultants is highly relevant for an accurate computation of the stress in beam elements undergoing large deformation.

\section{ACKNOWLEDGMENTS}

This work is part of the research programme HTSM 2017 with project number 16210, which is partly financed by the Netherlands Organisation for Scientific Research (NWO).

\section{REFERENCES}

[1] Wiersma, D., Boer, S., Aarts, R.G., Brouwer, D.M., Design and performance optimization of large stroke spatial flexures, Journal of computational and nonlinear dynamics, 9(1) (2014) 011016. doi: 10.1115/1.4025669.

[2] Naves, M., Brouwer, D.M., Aarts, R.G., Building Block-Based Spatial Topology Synthesis Method for Large-Stroke Flexure Hinges, Journal of mechanisms and robotics, 9(4) (2017) 041006. doi: $10.1115 / 1.4036223$

[3] Boer, S.E., Aarts, R., Brouwer, D.M., Jonker, J.B., Multibody modelling and optimization of a curved hinge flexure, In: The 1st joint international conference on multibody system dynamics, Lappeenranta, (2010)

[4] Naves, M., Aarts, R., Brouwer, D., Large stroke high off-axis stiffness three degree of freedom spherical flexure joint, Precision engineering, 56 (2019) 422-431. doi: 10.1016/j.precisioneng.2019.01.011.

[5] Brouwer, D.M., Meijaard, J.P., Jonker, J.B., Large deflection stiffness analysis of parallel prismatic leaf-spring flexures, Precision engineering, 37(3) (2013) 505-521. 
[6] Ugural, A.C., Stresses in beams, plates, and shells. CRC press (2009)

[7] Saje, M., Turk, G., Kalagasidu, A., Vratanar, B., A kinematically exact finite element formulation of elastic-plastic curved beams, Computers \& structures, 67(4) (1998) 197-214.

[8] Vratanar, B., Saje, M., A consistent equilibrium in a cross-section of an elastic-plastic beam, International Journal of Solids and Structures, 36(2) (1999) 311-337.

[9] Zupan, D., Saje, M., Finite-element formulation of geometrically exact three-dimensional beam theories based on interpolation of strain measures, Computer Methods in Applied Mechanics and Engineering, 192(49-50) (2003) 5209-5248.

[10] Sen, S., Beam Constraint Model: Generalized Nonlinear Closed-form Modeling of Beam Flexures for Flexure Mechanism Design. (2013).

[11] Jonker, J., Implementation of shear deformable thin-walled beam element for flexible multibody dynamics, In: Proceedings of the 8th ECCOMAS thematic conference on multibody dynamics 2017, Prague, Czech Republic, (2017)

[12] Nijenhuis, M., Meijaard, J., Brouwer, D., A spatial closed-form nonlinear stiffness model for sheet flexures based on a mixed variational principle including third-order effects, Precision Engineering, 66 (2020) 429-444. doi: 10.1016/j.precisioneng.2020.08.003.

[13] Nijenhuis, M., Jonker, B., Brouwer, D., Importance of warping in beams with narrow rectangular cross-sections: an analytical, numerical and experimental flexible cross-hinge case study, In: European Congress on Computational Methods in Applied Sciences and Engineering: Springer (2019)

[14] Vlasov, V.Z., Thin-walled elastic beams. Jerusalem: Israel program for scientific translations (1961)

[15] Cowper, G., The shear coefficient in Timoshenko's beam theory, Journal of applied mechanics, 33(2) (1966) 335-340.

[16] Dwarshuis, K., Aarts, R., Ellenbroek, M., Brouwer, D., Kinematically started efficient position analysis of deformed compliant mechanisms utilizing data of standard joints, Mechanism and Machine Theory, 152 (2020) 103911. doi: 10.1016/j.mechmachtheory.2020.103911.

[17] Trahair, N.S., Flexural-torsional buckling of structures. CRC press (2017)

[18] Timoschenko, S., On the torsion of a prism, one of the cross-sections of which remains plane, Proceedings of the london mathematical society, 2(1) (1922) 389-397.

\section{APPENDIX A - INCONSISTENCY INTERNAL SHEAR FORCE}

This appendix explains why an inconsistency is found between the shear force that is obtained based on the equilibrium-method and the shear force obtained by the constitutive law, even for short beams. The inconsistency occurs in generalized strain beam elements. First the relations of these elements are summarized. Then two causes of the inconsistencies are derived. Finally, it is shown why the error in the final stress is small in engineering practice.

The derivations in this appendix use the formulation of the $2^{\text {nd }}$ order element [11], but also hold for the used $3^{\text {rd }}$ order element, which are both generalized strain elements.

\section{Summary generalized strain beam formulation}

In a generalized strain beam element deformation modes are defined, which are related to the nodal coordinates

$$
\varepsilon=\mathcal{D}(x)
$$

The used beam elements have 8 deformation modes (eq. 7 of [11]), but only the first 6 are relevant. They are visualized in Fig. 7. 

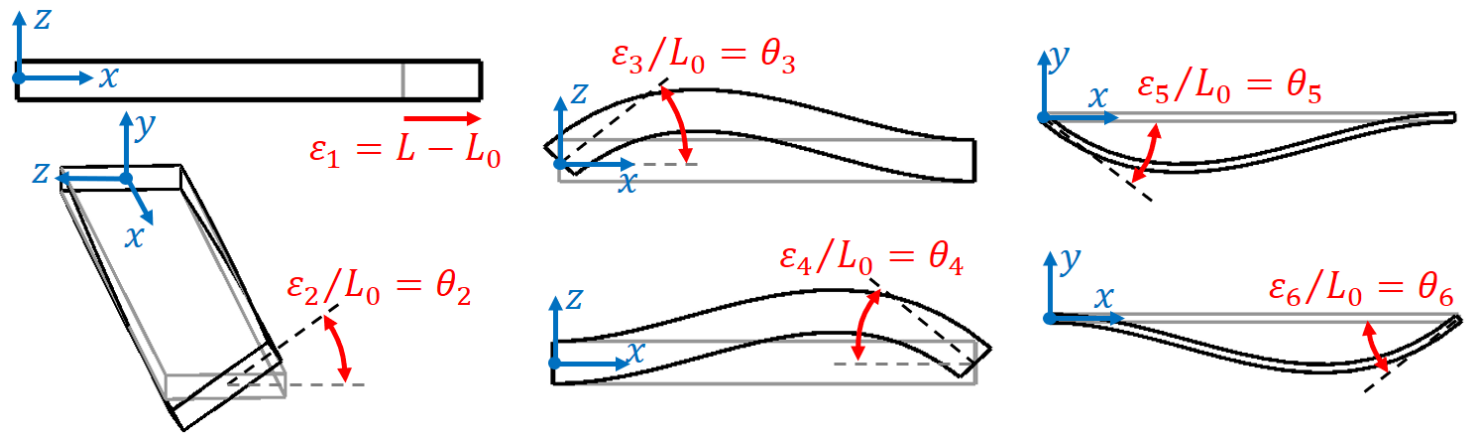

Figure 7. Six deformation modes of the beam element

The generalized forces of these deformation modes are called generalized external stresses, $\boldsymbol{\sigma}$. They are related to the generalized strains by a constant stiffness matrix: $\boldsymbol{\sigma}=\boldsymbol{S} \boldsymbol{\varepsilon}$. The exact expression for the stiffness matrix is given in eq. 40 of [11], but not relevant for the current derivation. According to the principle of virtual work the nodal forces are related to the generalized stresses (eq. 15 of [11])

$$
\boldsymbol{F}=\mathcal{D}_{, \boldsymbol{u}}^{T} \boldsymbol{\sigma}
$$

in which $\boldsymbol{F}$ consist of 12 terms, i.e. the forces and moments at both nodes. $\mathcal{D}_{, \boldsymbol{u}}^{T}$ is the derivative of $\mathcal{D}(\boldsymbol{x})$ to the nodal displacements and rotations, $\boldsymbol{u}$. The exact relations given in eq. 10-15 of [11]. The relevant results (eq. 16 of [11]) are:

$$
\left.\begin{array}{ll}
\sigma_{1}=-F_{x}^{p}=F_{x}^{q} & \text { (normal force) } \\
L \sigma_{2}=-M_{x}^{p}=M_{x}^{q} & \text { (torsion moment) } \\
L \sigma_{3}=-M_{y}^{p}, \quad L \sigma_{4}=M_{y}^{q} \\
L \sigma_{5}=-M_{z}^{p}, \quad L \sigma_{6}=M_{z}^{q}
\end{array}\right\} \text { (bending moments) }
$$

These reaction forces are also visualized in Fig. 8.

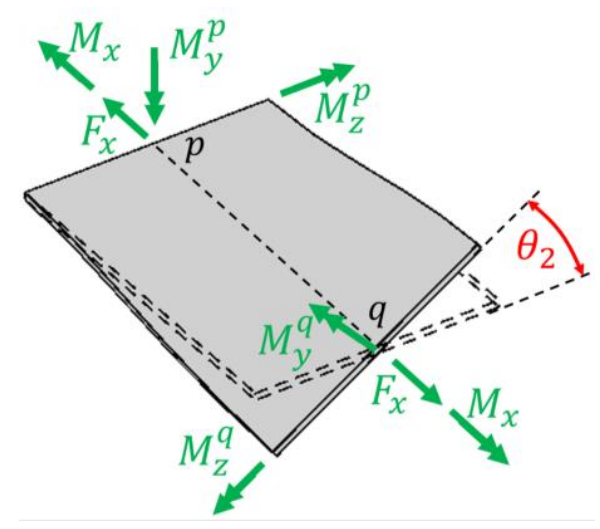

Figure 8. Reaction forces on a beam element with torsion

\section{Cause 1 - Different coordinate-axes}

In short the first reason for inconsistency is that the relations between the deformation modes and coordinates are defined using different coordinate axes such that also the relations between $\boldsymbol{\sigma}$ and reaction forces are defined in different axes. Fig. 8 shows for example that $M_{z}^{p}$ and $M_{y}^{p}$ have a different orientation. 
It can be easily seen from Fig. 8 that based on method A1 (equilibrium), the shear force at node $p$ should be:

$$
F_{z}^{p}=-\frac{M_{y}^{p}}{L}+\frac{M_{y}^{q}}{L} \cos \left(\theta_{2}\right)-\frac{M_{z}^{q}}{L} \sin \left(\theta_{2}\right)
$$

The same result is obtained by substituting eq. (13) into the full expression of eq. (12). By assuming a short beam element, such that also the deformations become small, we can linearize this result and substitute $\theta_{2}=\phi_{x}^{\prime} L$ :

$$
F_{z}^{p}=-\frac{M_{y}^{p}}{L}+\frac{M_{y}^{q}}{L}-\frac{M_{z}^{q}}{L} \phi_{x}^{\prime} L=-\frac{M_{y}^{p}}{L}+\frac{M_{y}^{q}}{L}-M_{z}^{q} \phi_{x}^{\prime}
$$

which is the internal shear stress at node $p$ that is found from equilibrium.

The resulting shear force at node $p$ based on method A2 (constitutive law) is intuitively only related to the moments around the $y$-axis:

$$
F_{z}^{p}=-\frac{M_{y}^{p}}{L}+\frac{M_{y}^{q}}{L}
$$

This result can also be obtained by a more detailed derivation: First substituting the mode shapes (eq. 38 of [11]) into eq. (3) of this paper:

$$
F_{z}^{p}=E A \kappa_{z} \cdot\left(u_{z}^{\prime}(s)+\phi_{y}(s)\right)=\frac{E A \kappa_{z}}{L} \cdot \frac{\Phi_{z}}{2\left(1+\Phi_{z}\right)}\left(\varepsilon_{4}-\varepsilon_{3}\right)
$$

where $\Phi_{z}=12 E I_{y} / G A \kappa_{y} L^{2}$. Then the inverse of the stiffness relation (eq. 40 of [11]) and eq. (13) can be used to obtain:

$$
F_{z}^{p}=\frac{1}{L}\left(-\sigma_{3}+\sigma_{4}\right)=-\frac{M_{y}^{p}}{L}+\frac{M_{y}^{q}}{L}
$$

The difference in result between the equilibrium method and the constitutive-law-method is the term $-M_{z}^{q} \phi_{x}^{\prime}$. This term can be nonzero, even for very short beams. As the bending moment in a very short beam is almost constant we will write this as $-M_{z} \phi_{x}^{\prime}$

\section{Cause 2 - Second order term in the deformation modes}

In the $2^{\text {nd }}$ order beam element a second order term is included which correct for the fact that the local rotation matrices are not linear in the virtual rotations around the $x, y$ and $z$ axis (eq. 45 of [11] gives the full expression of the rotation matrix). This causes a coupling term between the torsional deformation $\left(\varepsilon_{2}\right)$ and the bending deformation $\left(\varepsilon_{3}\right.$ till $\left.\varepsilon_{6}\right)$. This effect is included by modifying the strain definitions (see eq. 54 of [11]). The modification that causes an inconsistency is in the torsional mode:

$$
\hat{\varepsilon}_{2}=\varepsilon_{2}+\frac{1}{L}\left(-\varepsilon_{3} \varepsilon_{6}+\varepsilon_{4} \varepsilon_{5}\right)
$$

where $\hat{\varepsilon}_{2}$ is the second order generalized strain definition and the other generalized strains are the linear definitions as visualized in Fig. 7. For short elements, all the generalized strains become small, meaning that this second order term with squared generalized strains becomes negligible.

The reason for the inconsistency is that the extra term in the derivative $\widehat{\mathcal{D}}_{, \boldsymbol{u}}^{(2)}=d \hat{\varepsilon}_{2} / d \boldsymbol{u}$ does not become zero for short beams. This causes an extra term in eq. (12), which causes an inconsistency as derived in more detail below.

Eq. (12) can be evaluated for $F_{z}^{p}$. Note that $F_{z}^{p}$ is a term in $\boldsymbol{F}$ such that only the derivative of $\boldsymbol{D}$ to the corresponding displacement, $z_{p}$, is required:

$$
F_{z}^{p}=\widehat{\mathcal{D}}_{, z_{p}}^{T} \boldsymbol{\sigma}=\left(\frac{\partial \hat{\varepsilon}}{\partial z_{p}}\right)^{T} \boldsymbol{\sigma}=\frac{\partial \varepsilon_{1}}{\partial z_{p}} \sigma_{1}+\frac{\partial \hat{\varepsilon}_{2}}{\partial z_{p}} \sigma_{2}+\frac{\partial \varepsilon_{3}}{\partial z_{p}} \sigma_{3}+\frac{\partial \varepsilon_{4}}{\partial z_{p}} \sigma_{4}+\frac{\partial \varepsilon_{5}}{\partial z_{p}} \sigma_{5}+\frac{\partial \varepsilon_{6}}{\partial z_{p}} \sigma_{6},
$$

in which the nonzero terms in the derivative of $\hat{\varepsilon}_{2}$ are, see eq. (19): 


$$
\frac{\partial \hat{\varepsilon}_{2}}{\partial z_{p}}=\frac{\partial \varepsilon_{2}}{\partial z_{p}}-\frac{\varepsilon_{6}}{L} \frac{\partial \varepsilon_{3}}{\partial z_{p}}-\frac{\varepsilon_{3}}{L} \frac{\partial \varepsilon_{6}}{\partial z_{p}}+\frac{\varepsilon_{5}}{L} \frac{\partial \varepsilon_{4}}{\partial z_{p}}+\frac{\varepsilon_{4}}{L} \frac{\partial \varepsilon_{5}}{\partial z_{p}}
$$

In a beam element that is only deformed in bending around the $z$-axis, eq. (20) reduces to:

$$
F_{z}^{p}=-\left(\frac{\varepsilon_{6}}{L}+\frac{\varepsilon_{5}}{L}\right) \sigma_{2}-\sigma_{3}+\sigma_{4}=\left(\theta_{6}+\theta_{5}\right) \frac{M_{x}}{L}+\frac{M_{y}^{p}}{L}+\frac{M_{y}^{q}}{L}
$$

For short beam elements the resulting terms are:

$$
F_{z}^{p}=\frac{\theta_{6}+\theta_{5}}{L} M_{x}+\frac{M_{y}^{p}}{L}+\frac{M_{y}^{q}}{L}=\phi_{z}^{\prime} M_{x}+\frac{M_{y}^{p}}{L}+\frac{M_{y}^{q}}{L}
$$

This is the internal stress resultant found by method A1 (equilibrium). The internal stress resultant obtained by method A2 (Constitutive law) is given in eq. (16). So the inconsistent term is $\phi_{z}^{\prime} M_{x}$. This is a nonzero error, even for very short beam elements.

\section{Influence of the inconsistencies on the total stress}

The inconsistent terms in the shear stress are: $-M_{z} \phi_{x}^{\prime}$ and $\phi_{z}^{\prime} M_{x}$ as derived above. The resulting stress terms can be shown to be negligible for initially straight beams of common materials, based on classical beam theory, see e.g. [6]. For common materials we can assume that the maximum strain is limited to $1 \%$ and the maximum shear strain to $0.5 \%$, this limits the curvatures $\phi_{x}^{\prime}$ and $\phi_{z}^{\prime}$. For bending of a beam with rectangular cross section of thickness $t$ and width $w$ (Fig. 9) the relation between the highest strain and the bending curvature is:

$$
\varepsilon_{x x \text { max }}^{(\text {bend })}=\frac{t}{2} \phi_{z}^{\prime} \leq 0.01 \Rightarrow \phi_{z}^{\prime} \leq \frac{1}{50 t}
$$

For torsion, the relation between the highest shear stress and the curvature is approximately:

$$
\gamma_{x z \text { max }}^{(t o r)}=\phi_{x}^{\prime} t \leq 0.005 \Rightarrow \phi_{x}^{\prime} \leq \frac{1}{20 t}
$$

The following relations exist between the highest stress and the internal stress resultants of shear, bending and torsion:

$$
\tau_{x z \text { max }}^{(\text {shear })}=\frac{3}{2 w t} \cdot F_{z}(s), \quad \sigma_{x x \max }^{(\text {bend })}=\frac{6}{w t^{2}} M_{z}(s), \quad \tau_{x z \max }^{(\text {tor })}=\frac{6}{w t^{3}} T_{x}(s)
$$

Using these formulas we can relate the inconsistency in shear stress because of the terms $-M_{z} \phi_{x}^{\prime}$ and $\phi_{z}^{\prime} M_{x}$ to the existing stress of bending and torsion, accounting for the constraints in eqs. $(24,25)$ :

$$
\tau_{x z \max }^{(\text {shear,inconsis })}=\frac{3}{2 w t}\left|\phi_{z}^{\prime} M_{x}-M_{z} \phi_{x}^{\prime}\right| \leq \frac{3}{2 w t}\left|\frac{\tau_{x z \max }^{(\text {tor }} w t^{2}}{6} \cdot \frac{1}{50 t}-\frac{\sigma_{x x \max }^{(\text {bend }} w t^{2}}{6} \cdot \frac{1}{20 t}\right|
$$

By simplifying this equation we obtain the maximum extra shear stress:

$$
\tau_{x z \max }^{(\text {shear,inconsis })} \leq\left|\frac{\tau_{x z \max }^{(\text {tor })}}{100}-\frac{\sigma_{x x \max }^{(\text {bend })}}{80}\right|
$$

Which indicates that the error in shear stress is below $1 / 80$ of the total stress. Moreover, both terms in these equation are likely to partly cancel each other, which further reduces the error.

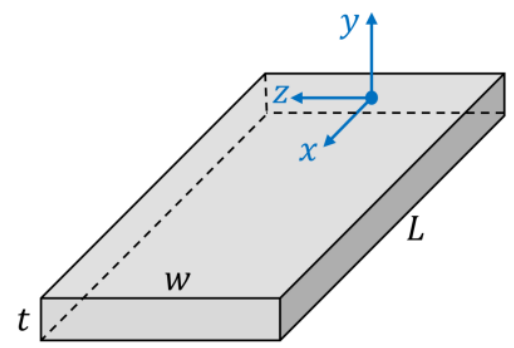

Figure 9. Rectangular cross section with thickness $t$ and width $w$ 